\title{
A randomised controlled trial of a cognitive- behavioural preventive intervention for the children of parents with depression: short-term effects on symptoms and possible mechanisms
}

Johanna Löchner ( $\nabla$ loechnerj@gmail.com )

Ludwig-Maximilians-Universität München: Ludwig-Maximilians-Universitat Munchen https://orcid.org/0000-0003-2886-9253

\section{Kornelija Starman-Wöhrle}

Ludwig-Maximilians-Universität München: Ludwig-Maximilians-Universitat Munchen Keisuke Takano

Ludwig Maximilians University of Munich: Ludwig-Maximilians-Universitat Munchen

\section{Lina Engelmann}

Ludwig-Maximilians-Universität München: Ludwig-Maximilians-Universitat Munchen

\section{Alessandra Voggt}

Ludwig Maximilians University Munich: Ludwig-Maximilians-Universitat Munchen

\section{Fabian Loy}

Ludwig-Maximilians-Universität München: Ludwig-Maximilians-Universitat Munchen

\section{Mirjam Bley}

Ludwig-Maximilians-Universität München: Ludwig-Maximilians-Universitat Munchen

\section{Dana Winogradow}

Ludwig-Maximilians-Universität München Max-von-Pettenkofer-Institut für Hygiene und Medizinische Mikrobiologie: Ludwig-Maximilians-Universitat Munchen Max-von-Pettenkofer-Institut fur Hygiene und Medizinische Mikrobiologie

\section{Stephanie Hämmerle}

Ludwig-Maximilians-Universitat Munchen

\section{Esther Neumeier}

Ludwig-Maximilians-Universität München: Ludwig-Maximilians-Universitat Munchen Inga Wermuth

Ludwig-Maximilians-Universität München: Ludwig-Maximilians-Universitat Munchen

\section{Katharina Schmitt}

Ludwig-Maximilians-Universität München: Ludwig-Maximilians-Universitat Munchen

\section{Frans Oort}

VU Amsterdam: Vrije Universiteit Amsterdam

\section{Gerd Schulte-Körne}


Ludwig-Maximilians-Universität München: Ludwig-Maximilians-Universitat Munchen

\section{Belinda Platt}

Ludwig-Maximilians-Universität München: Ludwig-Maximilians-Universitat Munchen

\section{Research article}

Keywords: prevention of depression, parental depression, family intervention, high-risk.

Posted Date: February 4th, 2021

DOI: https://doi.org/10.21203/rs.3.rs-162743/v1

License: (c) (i) This work is licensed under a Creative Commons Attribution 4.0 International License. Read Full License 


\section{Abstract}

Objective Parental depression is one of the biggest risk factors for youth depression. This parallel randomized controlled trial evaluates the effectiveness of the German version of the family-groupcognitive-behavioral (FGCB) preventive intervention for children of depressed parents.

Methods Families with i) a parent who has experienced depression and ii) a healthy child aged 8-17 years (mean $=11.63 ; 53 \%$ female) were randomly allocated (blockwise; stratified by child age and parental depression) to the 12-session intervention ( $\mathrm{EG} ; n=50$ ) or no intervention (CG; usual care; $\mathrm{n}=50$ ). Self-reported (unblinded) outcomes were assessed immediately after the intervention (6 months). We hypothesized that CG children would show a greater increase in self-reported symptoms of depression (DIKJ) and internalizing/ externalizing disorder (YSR/CBCL) over time compared to the EG. Intervention effects on secondary outcome variables emotion regulation (FEEL-KJ), attributional style (ASF-KJ), knowledge of depression and parenting style (ESI) were also expected. Study protocol (Belinda Platt, Pietsch, Krick, Oort, \& Schulte-Körne, 2014) and trial registration (NCT02115880) reported elsewhere.

Results We found significant intervention effects on self-reported internalising $\left(\eta_{p}^{2}=0.05\right)$ and externalising $\left(\eta_{\mathrm{p}}{ }^{2}=0.08\right)$ symptoms but not depressive symptoms or parent-reported psychopathology. Parental depression severity did not modify these effects. Both groups showed equally improved knowledge of depression $\left(\eta_{\mathrm{p}}{ }^{2}=0.06\right)$. There were no intervention effects on emotion regulation, attributional style or parenting style.

Conclusion The German version of the FGCB intervention is effective in reducing symptoms of general psychopathology. There was no evidence that the mechanisms targeted in the intervention changed within the intervention period.

\section{Introduction}

\section{Parental depression as a risk factor}

Depression is one of the most prevalent disorders and is predicted to be the world leading cause of illness by 2030 (WHO). One major risk for developing depression is having a parent who suffers from depression, which has been shown to be associated with adverse child outcomes such as increased internalising and externalising symptoms (Cummings \& Davies, 1994; Downey \& Coyne, 1990; Goodman \& Gotlib, 1999; Goodman et al., 2011; Weissman, Warner, Wickramaratne, Moreau, \& Olfson, 1997; Weissman et al., 2005) and a more severe and continuous course of depression (Goodman et al., 2011).

In the diathesis-stress model proposed by Goodman and Gotlib (1999) four mechanisms explain how children of depressed parents are at greater risk of depression: a) heritability, b) innate dysfunctional neuro-regulatory factors, c) exposure to negative maternal cognitions, behaviors, and affect, and d) the stressful context of the children's life. These are proposed to result in cognitive, affective and behavioural vulnerabilities in their offspring which reduce their ability to cope with stress. Affective vulnerability 
include maladaptive emotion regulation (ER) strategies (e.g. rumination, avoidance), which have been consistently shown to have an impact on the development of psychopathology (Schäfer, Naumann, Holmes, Tuschen-Caffier, \& Samson, 2016) and may play a mediating role in the transgenerational transmission of maternal depression (Silk et al., 2006). Moreover, adolescence goes along with using less adaptive but more maladaptive ER (Cracco, Goossens, \& Braet, 2017). Cognitive vulnerabilities include a negative cognitive style (e.g. the tendency to draw negative interpretations from ambiguous situations), which may be transmitted from parent to child (Dearing \& Gotlib, 2009; Dunbar et al., 2013; Hayden et al., 2014; Sfärlea et al., 2019). A number of preventive interventions have been developed for the children of depressed parents and have demonstrated that it is possible to reduce children's risk of depression (see Loechner et al., 2018 for a review and meta-analysis). Building on the model of Goodman and Gotlib (1999), one key component of preventive interventions involves building resilience to stress by strengthening children's cognitive, affective and emotional coping strategies using techniques from cognitive-behavioural therapy (CBT) (Clarke et al., 2001; Compas et al., 2009). A second component of preventive interventions is to improve family communication through psycho-education about depression (Beardslee et al., 1997; Compas et al., 2009; Mason, Haggerty, Fleming, \& Casey-Goldstein, 2012). By informing children about their parent's depression, they might be capable of understanding the adverse emotional reaction and behavior by their parents leading to an increased feeling of security and control (Hart, Newell, \& Olsen, 2003; Lenz, 2005; Stieglitz, 2002). A final component of preventive interventions is to provide parenting training. Depressive episodes have been shown to negatively impact parent-child interaction and strongly impair parenting (Lovejoy, Graczyk, O’Hare, \& Neuman, 2000; Reck, Nonnenmacher, \& Zietlow, 2016; Tronick \& Reck, 2009; Zietlow, Schlüter, Nonnenmacher, Müller, \& Reck, 2014). By improving parenting consistency preventive interventions may buffer against the effects of parental negative affect (Compas et al., 2009; Sanford et al., 2003).

Most existing preventive interventions for the children of depressed parents have focused on just one of the above components. For example, children's coping strategies (Clarke et al., 2001), communication within the family (Beardslee et al., 1997) or parenting training (Sanford et al., 2003). However, one intervention, the family group cognitive-behavioral (FGCB) preventive intervention for families of depressed parents (Compas et al., 2009), includes all three of the aforementioned elements. Compared to an active control group, the 12-session FGCB intervention was associated with reduced self-reported internalising symptoms immediately after the intervention ( 6 months from baseline) with a small effect size (ES) of $d=0.36$ (Compas et al., 2015). Effects on self-reported symptoms of depression and externalizing symptoms were small and only emerged 12-months after baseline (Compas et al., 2015). 24 months after baseline there was a very large positive effect of the intervention on incidence of depression (Compas et al., 2015).

Furthermore, as is the case for most preventive interventions for the children of depressed parents, the FGCB intervention was developed in the United States (U.S.) and has not been evaluated elsewhere. Coping with mental illness is culturally very different (Kirmayer, 2007; Perrino, Beardslee, \& Bernal, 2015), as is communication and parenting. A qualitative evaluation of the FGCB suggests that the intervention is accepted by families with depression in Germany (Claus et al., 2019). However, it remains unknown to 
what extent the FGCB is effective in modifying children's symptoms of psychopathology. Furthermore, it remains unknown whether the same factors which mediate and moderate the intervention in the U.S. also apply in Germany.

Since all existing interventions for the children of parents with depression show relatively modest effects which diminish over time (Loechner et al., 2018), understanding which intervention components are more or less effective is essential for the development of improved interventions. In the original evaluation of the FGCB around half of the improvements in symptoms from 0 to 12-months for those in the intervention group were mediated by changes in children's coping strategies and parents' parenting style from 0 to 6 months (Compas et al., 2010). Understanding which factors moderate the effects of interventions is important for the dissemination of interventions into routine practice. The most commonly studied moderator of interventions for children of depressed parents is the characteristics of parental depression when the family is enrolled in the intervention, e.g. whether the parent is depressed at baseline or not; how severely the parent is depression. These findings are heterogeneous: in one large trial, children whose parents were not depressed at baseline benefited more from the intervention than those whose parents were experiencing a current depressive episode (Beardslee et al., 1997; Brent et al., 2015; Garber et al., 2009). However, this finding could not be replicated in other trials (Clarke et al., 2001; Compas et al., 2009; Compas et al., 2015). A previous trial of the FGCB found moderating effects for a number of other variables such as child age, child gender and parental education (Compas et al., 2015), although it should be noted that these effects were found for some but not all follow-up points. Another trial found that lower levels of functioning, higher levels of hopelessness and elevated symptoms of psychopathology of the children at baseline reduced the effects of an intervention (Weersing et al., 2016) although these findings are yet to be replicated.

In sum, a major limitation of the existing literature is the lack of information on which specific components of existing interventions work and under which intervention settings.

\section{The current study}

The first aim of the current study is to perform the first evaluation of the FGCB intervention outside of the original research group, in another country and culture. We seek to replicate previous findings (Compas et al., 2015) that the FGCB is associated with less of an increase in self-reported internalising symptoms in children immediately after the intervention compared to a control condition. We also explore whether our adaptation of the intervention is effective in modifying self-reported externalising symptoms and depressive symptoms as well as parent-reported psychopathology. Secondly, we investigate whether mechanisms that are targeted in the intervention (ER, attributional style, parenting and knowledge of depression) improve in the intervention versus the control group. Thirdly, we explore whether children whose parents are not depressed at baseline may benefit more from the intervention. Finally, since parental well-being is associated with their children's symptomology (Keller \& Gottlieb, 2012), we investigate whether the intervention was associated with improvements in parents' symptoms of 
depression, as it had been in the original study 12- (Compas et al., 2009) but not 15- (Compas et al., 2011) months after the intervention.

\section{Method}

\section{Study design}

In a randomized controlled trial reported in line with the CONSORT statement (see Supplement 5), the FGCB intervention (Compas et al., 2009) was adapted to German ("Gesund und glücklich aufwachsen"; GuG-Auf) and evaluated. Psychiatrically healthy children and their parent(s) with depression were allocated to either the experimental group (EG) or to a usual care control group (CG). Figure 1 displays an overview of the study design. The randomisation procedure was performed by an independent researcher (FO) who was blinded to the identity of families. Randomisation was block-wise (per 10 recruited families[1]) and was stratified based on whether parents were currently (versus remitted) depressed and the age of children. JL and KS-W enrolled participants and provided lists of family ID numbers and information necessary for stratification (parental depression status, child age). BP matched families based on stratification factors and provided blocks of family ID numbers to FO. FO generated the assignment sequence via computer and allocated families to groups. JL and KS-W were informed about which group each family had been assigned to by BP. All families were assessed at baseline (T1), immediately after the intervention (T2; 6 months) as well as nine- (T3) and fifteen-months (T4) after baseline[2]. The sample size calculation was based on the primary outcome (episode of depression at T4) and assumed an effect size of $d=0.6$ which was based on previous studies finding an incidence of depression in 33\% of a no-intervention CG (Beardslee et al., 2013; Garber et al., 2009) and $10 \%$ of a EG who received the FGCB (Compas et al., 2015). In order to detect significant effects with an alpha level of $5 \%$ and power of $80 \%$ an a priori calculated one-sided Fisher's exact test for determining the necessary sample size revealed in a necessary $n=92$ (B Platt, Pietsch, Krick, Oort, \& Schulte-Körne, 2014). Although the sample was powered primarily to detect group differences in the onset of depression at $\mathrm{T} 4$, it assumed a medium ES of $d=0.6$. This ES is in line with other studies of the effects of preventive interventions on symptom change post-intervention (Compas et al., 2015; Judy Garber et al., 2009)

\section{Participants}

The 100 families ( $E G=50, C G=50$ ) with 135 children were included in the study if a parent fulfilled the DSM-IV diagnostic criteria of a depressive disorder during the children's lifetime and a child (aged 8-17, IQ>85) did not meet the diagnostic criteria for a psychiatric disorder in the present or past. Additional siblings who did not meet the study criteria (e.g. too young) were allowed to join intervention sessions provided they were not in crisis or had severe psychological symptoms. Participants had to be fluent in German. Parents were excluded if they suffered from alcohol or substance abuse, bipolar disorder, reported psychotic symptoms, had a personality disorder or a suicidal crisis. In case both parents were suffering from depression, both parents were entitled to receive the intervention. Families taking part in family-based therapy that might interfere with the intervention effects were excluded. Each family 
received $€ 25$ at the beginning and the end of the study period to compensate them for their time. All participants were informed about the study procedure, possible risks and gave their written consent for study participation. The study was approved by the Ethics Committee of the Medical Faculty at the Ludwig-Maximilian University (Study ID: 3-14). Families were recruited through public advertisements (32\%), psychiatric hospitals and psychotherapists (26\%), city council database (17\%), medical staff $(12 \%)$, community centres $(4 \%)$, previous studies $(3 \%)$, word of mouth $(3 \%)$, information evening $(2 \%)$ or unknown sources (1\%).

\section{Study drop-outs}

In total, 38 of 50 families randomised to the EG (76\%) completed the intervention. The remaining twelve (24\%) EG families dropped out after randomisation (but before the intervention began), largely due to the time constraints it would involve (8), or because the child had become affected by a psychiatric disorder themselves (1), although some did not give specific reasons (2) or could not be contacted (1). One family in the EG dropped out of the study after completing the intervention due to moving away from the area. Four of the 50 families randomised to the CG (8\%) dropped out of the study after randomisation largely for unknown reasons (3) but for one family because of marital disagreement about the risks of involving their children in a study about parental depression (1). One additional CG family (2\%) dropped out during the intervention period because the child no longer wanted to complete the questionnaires. Across both groups post-intervention data were sometimes unavailable without families formally dropping out of the study (see Missing Data).

\section{Sample description}

A description of the sample characteristics is provided in Table 1.66 families lived in Munich and 34 in surrounding areas. Most of the sample was German (91.5\%) and a minority (8.5\%) reported to have roots in Turkey, Croatia, Bulgaria and Austria. Families had a high socio-economic status and parents were mostly well educated (Starman, 2018)[3]. Five parents in the EG and eight parents in the CG were single parents. There was evidence that the groups differed in positive parenting at baseline indicating slightly more positive parenting skills in the EG $\left(t_{1,99}=-0.81, p=.039\right)$ and higher self-reported internalising symptoms in the EG $\left(t_{1,99}=2.04, p=.044\right)$. Taking a closer look, the group difference in internalising symptoms was driven by one outlier in the EG $(M=35)$, since there were no group differences when this one was excluded. The groups did not differ on any other variable ( $p s>.05)$.

\section{Psychopathology parents[4].}

Most parents were diagnosed with recurrent depressive disorder with mild $(64.5 \%)$ or moderate $(12.5 \%)$ severity and $23 \%$ were in remission. Ten percent fulfilled the criteria for a double depression. Only $14.8 \%$ had experienced a single depressive episode in their lifetime. $38 \%$ had comorbid diagnosis (mostly anxiety or eating disorders) $15 \%$ had slightly increased values on the personality disorder screening questionnaire (SKID II), but none showed clinically significant symptoms[5]. $11.5 \%$ of the families consisted of two parents suffering from depression. The partner of parents with depression that reported 
not to be affected by a mental illness was also screened for psychopathological impairment using the SCL-90-R indicating $11 \%$ with current symptoms of a psychiatric disorder.

\section{Procedure}

Families were recruited between July 2014 and October 2017. Follow-up data (T2) were collected between August 2015 and May 2018. Following a telephone-based screening, families were invited to the Department of Child and Adolescent Psychiatry at the Ludwig-Maximilian University to receive more detailed information about the study, provide consent and take part in clinical interviews (with parents and children separately). Questionnaires were given to take home and return within a week. Once 10 families were recruited, randomization procedure took place and the families were informed about which group they were allocated to. At the end of the intervention (6 months after baseline) families received questionnaires and were asked to send them back.

\section{Measures}

Table 2 gives an overview of the instruments used to determine eligibility for the study and outcome measures.

\section{Eligibility criteria}

Diagnostic status of parents and children. The semi-structured clinical interview Diagnostisches Interview für Psychische Störungen (Schneider \& Margraf, 2011) was administered to determine diagnostic status of parents according to DSM-IV. Interrater reliability is high $(\kappa=.72$ and $\kappa=.92)$ for general factors (Suppiger et al., 2008). The child version, that includes interviews of parents and child, was used to confirm that no child met the criteria for any mental illness (Unnewehr et al., 2009). In case parents and children reported differently, the child report was given greater weight since parents suffering from depression tend to underestimate the impact of child's potential symptoms (Angold et al., 1987). To assess reliability in the current sample twenty families were randomly selected and recordings of their interviews independently rated by LT or KM. The pre-defined criterion was whether the person was currently depressed or not. The accordance rate for parents $(n=15)[6]$ and children $(n=21)$ was excellent $(100 \% ; k=1.00)$.

Intelligence screening (child). The Culture Fair Test (CFT 20-R, Weiß, 2006) is an established multiplechoice intelligence assessment with five response options characterized by a very good re-test reliability $(r=.96)$ and correlates well with other intelligence tests (Weiß, 2006b). The CFT-20-R is split into two parts with four sub tests each (serial continuation series, object classification, matrix and topologies). In this study the short-version (part one; 56 items) was used.

\section{Outcome measures}

Symptoms of depression (child). The German version (DIKJ, Stiensmeier-Pelster et al., 2014) of the selfreport Children's Depression Inventory (CDI, Kovacs, 1992) contains 26-items scored on a 3-point Likert 
scale. The DIKJ has high internal consistency (Cronbach's alpha $a=.92)$ and good temporal stability (retest reliability $r t t=.76)$. Construct validity is high, given that the items are directly based on the DSMcriteria for depression, external validity is moderate (positive correlations with self-esteem $r=.60$ and anxiety $r=.61$ ) (Stiensmeier-Pelster et al., 2014). In addition to the DIKJ, the depression and anxiety scale of the Child Behavior Checklist (CBCL, Döpfner et al., 1994) and Youth Self-Report (YSR, Döpfner, Berner, et al., 1994) were used to measure depressive symptoms.

Children's internalizing and externalizing symptoms. German versions of the parent-report Child Behavior Checklist (CBCL, Döpfner, Schmeck, \& Berner, 1994) and its self-report equivalent the Youth Self-Report (YSR, Döpfner, Berner, \& Lehmkuhl, 1994) were used. They contain 123-items with 3- or 4-point Likert scales ( $0=$ "not at all" to $2=$ "exactly true"). Internal consistency for the internalising and externalising subscales of the CBCL $(r>$. 85) and YSR $(r \geq .86)$ is high.

\section{Secondary outcome variables}

Children's knowledge of depression. The "Wissensfragebogen Depression" (Allgaier, Schiller, \& SchulteKörne, 2011) assesses children's knowledge of the symptoms and treatment of depression using 50 items (e.g. "suffering from depression means that someone is crazy" or "Depression means to be sad for a long period of time") that are answered in a 4-point likert scale ( $0=$ "not at all true", ="completely true"). The questionnaire has not yet been validated.

Children's ER. The self-report questionnaire Fragebogen zur Erhebung der Emotionsregulation bei Kindern und Jugendlichen (FEEL-KJ; Grob \& Smolenski, 2005) assesses how children cope with the emotions anxiety, sadness and anger. It consists of 90 items that assess the use of adaptive (problem focused action, distraction, increased happiness, acceptance, forgetting, cognitive reappraisal, problem solving) and maladaptive (giving up, aggressive behavior, withdrawal, negative self-evaluation, perseveration) ER strategies. Each item (e.g. "When I'm angry, I keep my feelings to myself") is rated on a 5-point Likert scale according to how often it is applied ("never" to "almost always"). The internal consistency is good for the adaptive ( $a=.93)$ and maladaptive $(a=.82)$ ER subscales ("Fragebogen zur Erhebung der Emotionsregulation bei Kindern und Jugendlichen - FEEL-KJ.," n.d.) The six-week re-test-reliability for the two subscales is good $\left(r_{t t}=.81\right.$, adaptive strategies; $r_{t t}=.73$, maladaptive strategies) ("Fragebogen zur Erhebung der Emotionsregulation bei Kindern und Jugendlichen - FEEL-KJ.," n.d.). Sum scores for adaptive and maladaptive strategies were calculated for each emotion (clustering anger, anxiety and sadness).

Child attributional style. The Attributionsstil-Fragebogen für Kinder und Jugendliche (ASF-KJ; StiensmeierPelster, Schürmann, Eckert, \& Pelster, 1994)) consists of eight positive and eight negative situations whose causes are rated on a 4-point scale in three dimensions: external vs. internal ( $1=$ "caused by other person or circumstances" to $4=$ "I caused this event"), instable vs. stable ( $1=$ "will never be important" to $4=$ "will always be very important"), and specific vs. global ( $1=$ "is just this time relevant" to $4=$ "will also be relevant at other occasions"), resulting in a total of six subscales. Reliability (Cronbach's alpha) of the 
global and stability dimension lie between $\alpha=.72$ and $a=.81$ and the internality dimension between $a=.52$ and $a=.57$ (Stiensmeier-Pelster et al., 1994). Retest-Reliability (four weeks) was acceptable $\left(r_{t t}=.49\right.$ and $r_{t t}=$.65) (Stiensmeier-Pelster et al., 1994). We used sum scores of the three dimensions of positive and negative attributional scales. [7]

Parenting style. The parenting style inventory (ESI, Krohne \& Pulsack, 1991) is a 65-item child-report questionnaire covering positive (support, praise) and negative (criticism, restraint, inconsistency) parenting styles. Items are rated on a 4-point Likert scale ( 1 = "never or rarely happens" to 4 = "always happens"). The retest-reliability is modest (.51-.72), while the internal consistency of both the mother's and father's part is good (0.77-0.92 and 0.65-0.71) (Krohne \& Pulsack, 1991). Sum scores for positive (praise, support) and negative parenting (criticism, restraint, inconsistency) styles were calculated.

Parents' symptoms of depression. To test whether parents' symptoms of depression moderated the effects of the intervention we used the German version of the 21-item Beck's Depression Inventory (BDI-II, Hautzinger et al., 1994). The BDI-II has a 4-point Likert scale ( $0=$ "I do not feel sad" to $3=$ "I am so sad or unhappy that I can't stand it."). Test re-test (5 months) reliability is $r=.78$ (Hautzinger et al., 1994). Correlations with other measures of depression e.g. FDD-DSM-IV (Fragebogen zur Depressionsdiagnostik nach DSM IV, Kühner, 1997) are high ( $r=.72-.89)$.

Acceptance of the intervention Participants completed anonymous feedback questionnaires at the end of each session (Supplement 2). Parents and children were asked to rate on a 5-point Likert scale 1) whether they understood the content of the session ( $1=$ "not at all"; $5=$ "very well"), 2 ) whether they participated actively ( ="not at all"; $5=$ "a lot"), 3) whether they felt comfortable , 4) whether they felt supported and understood by the group leader, 5) how well they understood the homework assignment and 6) how helpful the session was (3)-6):1 ="not at all"; $5=$ "very much").

\section{Intervention}

GuG-Auf ("Gesund und glücklich aufwachsen") is the German adaptation of the FGCB (Compas et al., 2009). The manualized intervention (available upon request) is a group-, family- and CBT-based intervention combining parent-child and individual (parent and child only) sessions. The intervention lasts six months and includes eight weekly sessions and four monthly booster sessions designed to troubleshoot problems, families may be having in implementing the learnt skills into everyday life. The intervention contains three basic components: psycho-education of parental depression (parents and children), stress coping strategies for children's acceptance, distraction, positive thinking and positive activities [8]) and parenting training for parents (parenting and depression, displaying warmth and structure). Children and parents have homework tasks to complete between sessions and parents are encouraged to spend at least 15 minutes of quality time a week with their children.

The FGCB manual was translated into German by a bilingual member of the research team (LBW). Ambiguities were discussed with the authors of the original intervention. Some cultural adaptations were made to the manual including addressing parents using the formal "Sie" (children were addressed using 
the informal "du"), modifying leisure activities (e.g. football instead of baseball) and food examples (e.g. pretzels instead of crisps). In addition, more subtle cultural adaptations were made in order to match characteristics of the German culture [9].

Group leaders consisted of psychology doctoral students (KS-W, JL), trainee clinical psychologists (JL; LE, ED, KS, MB), or trainee child and adolescent psychiatrists (AV, IW, FL, SH). The majority of the sessions were conducted by JL (36\%), KS-W (35\%), LE (27\%), AV (21\%) and FL (20\%). Springers conducted a minority of sessions: MB (12\%), SH (9\%), ED (9\%), IW (8\%), KS (8\%). Occasionally undergraduate psychology students ran the groups when sickness did not allow otherwise: DW (12\%), MK (5\%), NC (3\%). All group leaders received training in the intervention by BP, JL and KS-W and regular supervision (every session) by the principle investigators (BP, GSK).

Eleven groups of 3-5 families were run between January 2015 and June 2018. The sessions took place in the Department of Child and Adolescent Psychiatry of the Ludwig Maximilian University Hospital.

Fidelity of intervention. To enhance treatment fidelity group leaders followed the GuG-Auf manual strictly. To evaluate treatment fidelity two independent assessors (AH, LD) viewed a sub-sample of sessions and completed an adherence checklist (available upon request). Of the 220 videotaped sessions (sessions four to 11 contained separate videos for child- and parent-sessions), 55 (25\%) were randomly selected, rewatched, and rated adherence. Ten of the 55 sessions had incomplete recordings of the full session (e.g. camera turned on too late) and these parts of the session were not included in analysis. The average rate of completeness of intervention characteristics was high: $98.0 \%$ of sessions were fully completed (range $87 \%$ to $100 \%)$.

Attendance and homework completion lists were kept for all groups but data were missing for group 11 . On average, families randomised to the intervention attended 7 sessions (range: 3 to 12). Of those families who attended at least one session, the average number of sessions attended was 9 and homework was completed by $69.79 \%$ of children and $60.63 \%$ of parents.

\section{Control condition}

The control group received no intervention but could receive support from the usual care system (e.g. parent advice centres, general practitioner; GP) and was offered the intervention in some form after the study period. The no intervention condition was chosen to compare the intervention with existing provisions. Families anecdotally reported receiving support from their GP or advice centres although this was not systematically assessed.

\section{Analysis strategy}

The data was analyzed using SPSS Version 19 (SPSS Inc., 1989-2006) for Windows and JASP Version 0.8.1.1. for Mac OX. An intention to treat approach was taken. T-tests on between-group ( $E G, C G)$ differences in the various outcome and confounding variables at baseline to check whether randomization was successful. 
Confounding variables such as child age and gender were intended to be included in analysis models. However, since neither variable was associated with changes in the outcome variables (all $p s>05$ ) we ran repeated-measures ANOVA tests on each of the outcome variables[10] including the oldest eligible child in the family since risk of depression increases across adolescence. This also allowed us to test main effects of time in order to explore whether the intervention had modified the mechanisms it proposed to target, similar models were also run for the variables iv) knowledge of depression, v) attributional style, and vi) ER. The intervention effect on parental depression was analysed in the same way as the main outcomes. In addition, we tested the moderating effect on the outcome variables by including the dichotomous covariate of parental depression at baseline (being currently depressed yes/no) that was assessed in the clinical interviews with each parent.

Acceptance of the intervention was descriptively analysed by calculating mean scores from the feedback questionnaires completed after each session (Supplement 2). Semi-structured interviews were conducted with a sub-set of families after the intervention and are reported elsewhere (Claus et al., 2019).

\section{Missing Data}

The range of missing outcome values varied from 10\% (BDI-II T1) to 58.0\% (DIKJ T2; Supplement 4). Importantly, the EG and CG groups did not differ significantly in the amount of missing data at baseline $\left(\mathrm{t}_{1,99}=.06 ; p=.415\right)$ or post-assessment $\left(\mathrm{t}_{1,99}=.17 ; p=.730\right)$. Thus, we assumed MAR (Allison, 2012) but due to the amount of missing data in the whole sample not MCAR. For the main analysis, missing values were imputed based on the expectation-maximization method (Stephens, Smith, \& Donnelly, 2001).

[1] Occasionally varying between 8 and 12 families

[2] Since data analysis is ongoing for T3 and T4 these data are not reported here.

[3] SES data were checked by the Winkler Index (Starman, 2018)

[4] Data refers to primary parent participating in the study

[5] One family was excluded because the parent had clinically-significant values on the SKID-II.

[6] 5 parent interviews were not successfully recorded.

[7] Since the six subscales of the ASF-KJ are each composed of only 8 items, they may indirectly affect the power of the analysis.

[8] in German: Akzeptanz, Ablenkung, positives Denken, positive Aktivitäten 
[9] Overly positive statements came across to German members of the team as being unauthentic and whilst group leaders were encouraged to be supportive of families they often refrained from using "we" to highlight families' own responsibility for their actions.

[10] The planned analyses reported in the study protocol involved linear regression models for i) child self-reported (YSR) and ii) parent-reported (CBCL) internalizing, externalizing and anxiety/depression symptoms as well as iii) children's self-reported depressive symptoms (DIKJ) (Platt et al., 2014).

\section{Results}

Figure 1 provides an overview of the participant flow within the trial.

\section{Intervention effects on symptom severity}

Table 3 provides means and standard deviations for each of the symptom severity variables (DIKJ, CBCL, YSR) before and after the intervention. Since the outcomes of the repeated-measures ANOVA test of group differences are reported in Table 3 (and supplement 1), they are not repeated in the text below.

\section{Self-reported symptoms of depression (DIKJ)}

Although both groups showed significant reductions in depressive symptoms over time $\left(F_{1,98}=5.78, p=\right.$ $\left..018, \eta_{p}^{2}=0.06\right)$, there were no significant differences between groups $\left(F_{1,98}=2.49, p=.118, \eta_{p}^{2}=0.02\right)$. Of note, we analysed anxiety-depression sub-scale of the YSR and found a significant intervention effect $\left(F_{1,98}=8.58, p=.004, \eta_{p}^{2}=0.08\right)$. Children in the EG reported significantly less symptoms over time ( $m=$ $1.94, S D=5.69, t=2.41, p=.019)$, while children in the CG showed more symptoms over time $(m=-1.02$, $S D=4.00, t=-1.77, p=.083)$. Parents in both groups reported significantly less anxiety-depressive symptoms over time $\left(F_{1,98}=3.97, p=.049, \eta_{p}{ }^{2}=0.04\right)$ but there were no group differences in change over time. These results were not modified by parental depression at baseline (all $p$ 's $>.05$ ).

\section{Self-reported internalizing and externalizing symptoms}

There was a significant difference between groups ( $E G$ vs. $C G)$ on changes in self-rated internalizing $\left(F_{1,98}=5.42, p=.022, \eta_{p}^{2}=0.05\right)$ and externalizing symptoms from T1 to T2. The EG showed significantly less internalising symptoms from T1 to T2 $(m=1.95, S D=6.13, t=2.25, p=.029)$ whereas the CG did not change over time $(p>.05)$. The EG showed no significant change in externalising symptoms over time $(p>.05)$ whereas the CG showed an increase $(m=-1.69, S D=5.19 \mathrm{t}=-2.31, p=$ .025). These results were not modified by parental depression at baseline (all $p s>.05$ ).

\section{Parent-reported child internalizing and externalizing symptoms}

There were no significant differences between groups $(E G, C G)$ in changes in parent-reported internalising or externalising symptoms from $\mathrm{T} 1$ to $\mathrm{T} 2$. These results were not modified by parental depression at baseline (all $p s>.05)$. 


\section{Effects of potential mechanisms}

\section{Emotion regulation (ER)}

There were no significant differences between groups $(E G, C G)$ in changes in adaptive or maladaptive ER. These results were not modified by parental depression at baseline (all $p$ 's $>.05$ ).

\section{Attributional style}

Both the EG $(m=-3.15, S D=9.12, t=-2.44, p=.018)$ and CG $(m=-4.37, S D=7.87, t=-3.92, p=.000)$ showed significantly more positive attributions over time $\left(F_{1,98}=19.47, p=0.000, \eta_{p}^{2}=0.166\right)$ but there was no difference between the groups. In addition, both the EG $(m=-6.27, S D=10.42, t=-4.26, p=.000)$ and CG $(m=-4.92, S D=7.87, t=-4.42, p=.000)$ showed increased negative attributional thinking $\left(F_{1,98}=\right.$ $\left.36.76, p=0.000, \eta_{p}{ }^{2}=0.273\right)$ but there was no difference between the groups $\left(F_{1,98}=0.54, p=0.465, \eta_{p}{ }^{2}\right.$ $=0.005$ ). These results were not modified by parental depression at baseline (all $p s>.05$ ).

\section{Parenting Style}

There was no evidence of group differences in changes in positive $\left(F_{1,98}=0.96, p=0.330, \eta_{p}^{2}=0.001\right)$ or negative $\left(\mathrm{F}_{1,98}=1.860, p=0.175, \eta_{p}{ }^{2}=0.019\right)$ parenting style. These results were not modified by parental depression at baseline (all $p s>.05$ ).

\section{Knowledge of Depression}

Although both groups showed more knowledge of depression over time $\left(F_{1,98}=45.97, p<.000, \eta_{p}{ }^{2}=\right.$ 0.32; $E G: m=-2.42, S D=4.06, t=-4.21, p<.001 ; C G: m=-2.71, S D=3.47, t=-5.51, p<.001)$, there was no evidence that this differed between the groups $\left(F_{1,98}=0.14, p=0.704, \eta_{p}^{2}=0.001\right)$ and modified by parental depression at baseline (all $p s>.05$ ).

\section{Parental depression}

Parents in both groups showed a significant reduction in depressive symptoms from T1 to T2 $\left(F_{1,98}=\right.$ $\left.15.75, p=.000, \eta_{p}^{2}=0.138\right)$, but did not differ from each other $\left(F_{1,98}=0.17, p=.677, \eta_{p}^{2}=0.002\right)$.

\section{Acceptance of intervention}

Descriptive ratings of the intervention on six scales according to parents and children are provided in the supplement 2 and 3 . Averaging across all sessions, parents provided a mean rating of 4.35 of a possible $5(S D=0.71)$ and children's ratings were similar $(M=4.35, S D=0.83)$.

\section{Discussion}


This RCT aimed to evaluate the effectiveness of GuG-Auf: the German adaptation of the family-and group-based cognitive behavioural (FGCB) intervention for the children of depressed parents (Compas et al., 2009). The original intervention showed positive effects in modifying self-reported internalising symptoms immediately after the intervention (Compas et al., 2015). This study is the first to attempt to replicate these effects outside of the USA and in an independent research group.

\section{Summary of findings}

As expected, there was a significant effect of the intervention on children's self-reported internalizing symptoms with a medium to large ES: children in the CG showed no change over time whereas children who received the intervention showed a decrease over time. Similar findings (this time a large ES) were also observed for children's self-reported externalizing symptoms: this time children in the CG showed an increase over time whereas children in the EG showed no change. Contrary to expectations, there was no effect of the intervention on children's self-reported depressive symptoms or parent-rated child psychopathology. The intervention had no effects on the proposed target mechanisms: although the EG showed significant (and very large) improvements in their knowledge of depression, so did the CG. Parental depression severity reduced in both groups over time with a large effect. Baseline parental depression severity did not have an impact on any of the outcome variables. The acceptance of the intervention was good.

\section{Effects of the intervention on symptom severity}

The finding that children in the EG showed improved internalizing symptoms whereas children in the CG showed a worsening of symptoms over time is in line with other studies of preventive interventions for children of depressed parents (Beardslee et al., 1997; Clarke et al., 2001; Compas et al., 2015; Garber et al., 2009; Loechner et al., 2018; Sanford et al., 2003). A meta-analysis of preventive interventions for children of depressed parents found a small ES for internalizing symptoms (Loechner et al., 2018). The ES in the current study is small-to-medium $\left(\eta_{p}{ }^{2}=0.05\right.$ is equivalent to $\left.d=.46\right)$ but nevertheless larger than the meta-analysisand in the original study (Compas et al., 2015). Our finding that the intervention was associated with improvements in externalizing symptoms contrasts with Compas et al., (2015), who found effects on externalizing symptoms emerged only at 12 months post-randomization.

The lack of intervention effects on depressive symptoms were unexpected and contrasts with the finding that according to the anxiety/depression sub-scale of the YSR, children in the EG showed a greater improvement of symptoms compared to the CG. The lack of effects on the DIKJ may be due to its poor psychometric properties ${ }^{11}$. In the current sample its reliability was questionable. In comparison, the reliability of the anxiety/depression scale of the YSR was good. Note that DIKJ scores did not correlate with the anxiety/depression sub-scale of the YSR at T2. An alternative explanation for the lack of effects on the DIKJ is that intervention effects only emerge later on in time. Indeed, Compas et al., (2015) found that intervention effects on depressive symptoms only emerged 12-months after baseline. Although some trials of preventive interventions for the children of depressed parents have found effects on depressive 
symptoms immediately after the intervention (Garber et al., 2009; Punamaki et al., 2013) the majority have not (Clarke et al., 2001; Compas et al., 2009, 2011, 2015; Sanford et al., 2003). One explanation for these delayed effects is that children have to be confronted with a certain amount of stress in order to be able to apply their coping skills.

We did not find significant effects of the intervention on parent-reports of children's internalizing or externalizing symptoms. This may be because self-reports of internalizing symptoms tend to show higher reliability than parent-reports (Schneider et al., 2013), especially if the parent is suffering from an acute episode of depression (Angold et al., 1987). Parents and children often perceive children's psychopathological symptoms differently (Choudhury et al., 2003) and in the current sample only small to moderate correlations were found between parent- and child-reported psychopathology (internalizing symptoms $r=.527^{*}$; externalizing symptoms $r=.472^{\star *} ; 47^{\star *}$ and anxiety/depression $\left.r=.09\right)$. Since depression is associated with reduced levels of empathy (O'Connor et al., 2002) and an increased selffocus (Morris \& Kanfer, 1983) parents in the current study may have been less sensitive to changes in their children's emotions and behavior n(Angold et al., 1995; Messer, Angold, Costello, \& Loeber, 1995; Orchard, Pass, Marshall, \& Reynolds, 2017). The finding that intervention effects were stronger according to child versus parent reports also replicates that of the original trial (Compas et al., 2015) as well as other prevention trials (Clarke et al., 2001).

\section{Potential mechanisms for the intervention}

We found no positive effects of the intervention on any of the hypothesized mechanisms. For example, we found no effects of the intervention on children's ER whereas the original trial did (Compas et al., 2010). One possible explanation for these findings is the different measures used between the two trials (the original trial used the Responses to Stress Questionnaire; RSQ). However, since our measure of ER (FEEL-KJ) included the ER strategies taught in the intervention (acceptance, distraction, positive thinking, positive activities) it is still surprisingly we did not find any positive effects. It is possible that a change on cognitive and behavioral factors over a relatively short period of time might be optimistic and more realistic in the later follow-up assessments.

In contrast with Compas et al., (2015) and our hypotheses, we also found no evidence that the intervention modified parenting style. These null-findings may also reflect measurement issues, since we assessed parenting style by children's reports whereas in the original trial (Compas et al., 2010) observations of parent-child interactions were used, which may have been more accurate.

Similarly, to most trials in this field (Beardslee et al., 1997; Garber et al., 2009; Punamaki et al., 2013) children in the EG showed improved knowledge of depression. However, in contrast to earlier studies, we also found children in the CG showed significantly improved knowledge of depression. This may be because the CG nevertheless received a 2-3 hour diagnostic session at baseline as well as birthday and Christmas cards and reminders to complete questionnaires (to maximize data completion). Improvements in children's knowledge of depression might also be due to the fact that families sought out extra psycho-educative support after being allocated to the CG. In addition, the process of enrolling 
their family in the intervention may have improved parents' communication and interaction with their children such that they were able to open up about their mental illness. Feeling more informed about their parents' illness might have increased feelings of control and security which may explain in part the internalizing symptom reduction (Lenz, 2009). Indeed, similar findings have been observed in other trials

of preventive interventions for the children of depressed parents (Punamaki et al., 2013; Solantaus et al., 2010).

\section{The moderating role of parental depression severity}

In contrast with positive findings from other preventive trials for the children of depressed parents (Beardslee et al., 1997; Brent et al., 2015; Garber et al., 2009) we did not find any evidence that parent's depression status (whether they were in an episode of depression at enrollment or not) moderated children's response to the intervention. It is possible that our failure to find moderating effects was due to the relatively short follow-up time ( 6 months). However, the original trial found no evidence that parental depression severity moderated children's outcomes at any of the time points ( 2 to 24 months; Compas et al., 2009, 2015). The original trial did not distinguish between parents with versus without a current episode of depression. Meta-analyses with large numbers of RCTs are needed to address the moderating role of parental depression status and/or severity.

Finally, the finding that parents in both groups showed improved depressive symptoms over time contrasts with findings from the original study, in which only those in the EG improved (Compas et al., $2009,2015)$. This supports the notion that families in the CG sought out their own support.

\section{Acceptance of the intervention}

The acceptance of the intervention was rated as good by the participants. The content appeared to be adequate and families felt understood and appreciated. These findings may explain the fact that none of the families in the EG dropped out during the intervention. This finding is crucial and especially relevant for clinical practice and the potential implementation of prevention. Note that 12 families dropped out between randomization to the EG and the start of the intervention, suggesting some aspects of the intervention may have put families off their initial intentions to participate. Unfortunately, we do not have any data on individual drop-out reasons.

\section{Strengths}

A major strength of the study was use of standardized clinical interviews (rather than self-reported questionnaires) to characterize parents and children. This increases the validity of the findings and was also intended to maximize ES (including parents who had sub-threshold levels of depression or children with elevated symptoms of depression may have reduced the effects). It is noteworthy that the questionnaire-based outcome variables at the 6-month follow-up are only proxy for depression prevention: the extent to which the intervention has a preventative effect can only be detected by analyzing data on the onset of depression at 15 months (data analysis ongoing). We also sought to 
include both parent and child measures, although it should be acknowledged that all measures were subjective reports which are nevertheless open to bias.

Another strength is the high level of treatment fidelity achieved, which likely reflects the highly manualized intervention, regular supervision and high levels of attendance (which were comparable to the original study; Compas et al., 2015). High treatment fidelity allows more valid attribution of positive findings to the intervention itself.

A final strength of the study is that this study represents the first replication of the FGCB intervention, which was originally developed in the U.S. and has not been replicated outside of the research group. Many psychological interventions are evaluated by those who developed them, who are naturally biased to demonstrate the efficacy of their intervention. The findings in the current study suggest the positive findings found by Compas et al. (2015) are not strongly influenced by researcher bias.

\section{Limitations}

An important limitation is the relatively high level of missing data (up to $51 \%$ in the $C B C L$ at $T 2$ ), which we had not anticipated. Although numerous measures were taken to obtain data at the three time points, families reported extremely high levels of strain which hindered them. The missing data limits the statistical power of the study, since the sample size calculation $(n=92)$, which was based on the primary outcome of onset of depression at 15-months, assumed an ES of $d=.6$ (power $=.80$, alpha $=.05)$. Since participants who completed the questionnaires were probably under less strain than those who did not, the data may be biased. It seems plausible that families under more strain may have benefited more from the intervention, which would suggest our data underestimate the true effect. It should be noted that it is hard to tell how our amount of missing data compares with other studies, since many do not report the amount of missing data (Compas et al., 2015).

One (uncontrollable) limitation to the external validity of the current study is the poor representativeness of the sample in terms of high socio-economic status, high levels of motivation and a lack of cultural diversity. Families with low socio-economic background are often more affected by depression and may face more stressors (e.g. financial problems, unemployment) and it is unclear if they would benefit equally from the intervention. The reason for our biased sample might be that families from low socioeconomic backgrounds have less time to participate in a time-consuming intervention due to work commitments or household tasks. Our sample of parents may also have been more motivated and prepared to open up about their depression than the average family experiencing depression. Reasons families reported for not wanting to participate included fears and shame about opening up about their diagnosis or overburdening their children with difficult topics. This was found in interviews with parents who declined to participate in a similar preventive intervention (Pihkala \& Johansson, 2008). Parents often may try to hide their illness due to fears about a possible loss of custody (Hearle et al., 1999). Families that participated were mostly German and as such the findings are also less representative for families from ethnic minorities. The importance of "scientific equity" across cultures and within societies when it comes to youth prevention is emphasised by Perrino et al., (2015). 
Another aspect which limits the interpretation of the findings is the relatively wide age range of the children included. We set the inclusion criteria to 8-17 years in order to maximise chances of recruiting the necessary sample size in the project time period and because children younger than 8 would not have the cognitive and emotional skills to take part in the intervention. Although this heterogeneity of the children means children differed in how recently they had been exposed to their parents' depression, we did at least require the depression to have been within the child's lifetime. Furthermore, the majority of parents had experienced multiple episodes of depression during the child's lifetime (a single post-natal episode was uncommon). Although including children above the age of typical onset of depression (15 years) may have led us to over-sample for particularly resilient children, in reality the average age of participants was 12 years and the majority $(x \%)$ were aged 9-15 years.

Finally, the internal validity of the study findings is also limited because we did not include an active CG. We included measures of possible mechanisms but an active CG would be necessary to know whether the findings might instead be attributed to "common factors" (e.g. the group setting) (Huibers et al., 2014). We decided to include a no-intervention CG because our primary aim was to evaluate the benefit of a preventive intervention compared to existing care. Indeed, with this study design we were able to show how rapidly some outcome variables (e.g. self-reported externalizing symptoms) worsened in the CG within just six months. This supports longitudinal studies of negative outcomes in children of parents with depression (Weissman et al., 2006).

\section{Future research}

In order to reduce the bias towards a particularly motivated sample with a high socioeconomic status (SES), future studies of prevention interventions may need to consider how they can reduce the stigma and financial costs associated with preventive interventions. Digital interventions administered in a stepped-care approach could be a bridge until families have the courage to participate in face-to-face interventions. Digital interventions may also be more attractive for families who live more remotely. Future research could also minimize the strain of data collected on participants by using digital platforms. Future studies could benefit from the inclusion of more objective assessments measures, for example: observations of parent-child interaction (Compas et al., 2010), physiological measures of stress reactivity (cortisol, electro dermal activity, heart rate) and ecological momentary assessment (EMA). Alternative therapeutic approaches such as interpersonal therapy (IPT) which have shown to be effective in the treatment (Zhou et al., 2015) and universal prevention (Young et al., 2010) (Young et al., 2010) of youth depression, may also show promise in the selective prevention of depression in the children of depressed parents. In general, more research and replications of existing trials (outside the U.S.) is needed to achieve more homogenous findings of how preventive interventions should be designed and implemented.

\section{Clinical implications}

The study finding have numerous implications for clinical practice and research. Firstly, they suggest that the intervention is acceptable for affected families in Germany. They also provide preliminary evidence to 
support the robustness of the original study findings: at least in the short-term (immediately after the intervention) the effects on symptom severity could be replicated. Secondly, the effect sizes associated with the intervention are relatively high compared to other prevention programs (Hetrick et al., 2016) (Hetrick et al., 2015). Although the intervention might be more time consuming than others, it is likely to be a worthwhile intervention investing in if the burden of depression is to be reduced. Finally, the high SES of the sample recruited highlights the need to consider how such interventions can be tailored to appeal to and reach families from less privileged backgrounds.

\section{Conclusion}

This is the first study to evaluate the effects of the FGCB preventive intervention for children of parents with depression outside of the original research group (Compas et al., 2009, 2015). Immediately after the intervention we found medium to large intervention effects for children's self-reported internalizing, externalizing and anxiety-depressive symptoms but no effect on parent-reported psychopathology. There was no evidence that the intervention effects were moderated by whether or not parents were in an episode of depression at baseline. In contrast to our expectations both groups showed increased knowledge of depression and decreased parental depression over time. Furthermore, we did not find effects of the intervention on potential mechanisms. Future evaluations of preventive interventions should consider ways of designing interventions which appeal to a more representative sample of children of depression parents.

\section{List Of Abbreviations}

FGCB: $\quad$ Family-Group-Cognitive-Behavioral prevention program

GuG-Auf: Gesund und Glücklich aufwachsen - translated and adapted FCGB to Germany

EG: $\quad$ Experimental Group

CG: $\quad$ Control group

ES: $\quad$ Effect Size

ER: $\quad$ Emotion Regulation

DIKJ: Depressionsinventar für Kinder und Jugendliche, Self-reported symptoms of depression

YSR Youth Self-Report Form; Self-reported psychopathology symptoms

CBCL: $\quad$ Child Behavior Checklist, Parent-reported child psychopathology symptoms

ESI: $\quad$ Erziehungsstil-Inventar, Parenting style inventory

ASF: $\quad$ Attributionsstil-Fragebogen für Kinder und Jugendliche, Child attributional style 
FEEL-KJ: Fragebogen zur Erhebung der Emotionsregulation bei Kindern und Jugendlichen, Emotion Regulation in Children

BDI-II: $\quad$ Beck's Depression Inventory, Depressive Symptoms Parents

\section{Declarations}

\section{Ethical approval}

We hereby declare that this study was approved by the Ethics Committee of the Medical Faculty at the Ludwig-Maximilian University (Study ID: 3-14).

\section{Consent for publication}

All participants were informed about the study procedure, possible risks and the publication of the study results. All participating families (parents and children) gave written informed consent to participate in the study.

\section{Availability of data and materials}

All data and materials can be provided upon request by the last author (Belinda Platt). We have no conflict of interest. Data transparency statement: We are happy to share an anonymized data set upon request.

\section{Competing interests}

The authors declare that they have no competing interests.

\section{Funding}

The present study was supported by the "gesund.leben.bayern", the "Förderprogramm für Forschung und Lehre" (FöFoLe; Reg.-Nr. 895) of the Medical Faculty of the Ludwig-Maximilians-University Munich, the "Hans und Klementia Langmatz Stiftung" as well as the Gender Mentoring Program of the LudwigMaximilians-University Munich.

\section{Authors' contributions}

$\mathrm{JL}, \mathrm{KSW}$ and LE conducted the study design and were involved in all study procedures (including assessment, recruitment, group management, data collection and analysis). BP and GSK were the Principal Investigators and supervised the team. JL wrote the manuscript under supervision of BP and with contributions to the introduction from KSW, GSK, SH, LE, EN, DW, and MB. FO and KT supported the data analysis by providing regular advice on the evaluation processes. AV, FL, IW and KS provided substantial feedback on the entire manuscript and contributed comments to the introduction and discussion of the main author team. 
All authors read and approved the submitted version. We hereby declare that all authors have agreed to be personally responsible for their contributions and to ensure that questions regarding the accuracy or integrity of any part of the work can be answered.

As corresponding author, I declare, that no changes of authorships have been conducted and all authors deserve authorship due to their substantial contribution to the study and manuscript.

\section{Acknowledgements}

We thank all participating families for the time and effort, they spent to participate and support the project. In addition, we thank all research assistands and group leaders, listed here

Research assisstants (clinical interviews, data collection, recruitment): Petra Wagenbüchler, Kirsten Moser, Carolina Silberbauer, Veronika Jäger, Nathalie Claus, Lina Engelmann, Lisa Ordenewitz, Angelina Mooseder, Dana Winogradow, Moritz Dannert, Ann-Sophie Störmann, Jakob Neumüller.

Group leaders of the intervention: Lina Engelmann, Alessandra Voggt, Fabian Loy, Mirjam Bley, Dana Winogradow, Stephanie Hämmerle, Esther Neumeier, Inga Wermuth, Katharina Schmitt

\section{Author Information}

Johanna Löchner is a licensed clinical psychologist (cognitive behavioral therapy) and holds a PhD in psychology. Since 2012 she works in both fields, clinical practice working with adults/parents, children and adolescents as well as in research. Her research focusses on risk factors and transmission of prevention of mental illness, with a strong approach to enhance evidence-based intervention for high-risk population in to clinical practice. Since 2020, she leads her own research group in the German Early Prevention Center at the German Youth Institute, that is well connected to politics and the practical field of families.

\section{References}

Allgaier, A.-K., Schiller, Y., \& Schulte-Körne, G. (2011). Wissens- und Einstellungsänderungen zu Depression im Jugendalter. Entwicklung Und Evaluation Einer Aufklärungsbroschüre, 20, 247-255.

https://doi.org/10.1026/0942-5403/A000062

Allison, P. D. (2012). Handling Missing Data by Maximum Likelihood. SAS Global Forum 2012 Statistics and Data Analysis, 1-21.

Angold, A., Weissman, M. M., John, K., Merikancas, K. R., Prusoff, B. A., Wickramaratne, P., ... Warner, V. (1987). Parent and Child Reports of Depressive Symptoms in Children At Low and High Risk of Depression. Journal of Child Psychology and Psychiatry, 28(6), 901-915. https://doi.org/10.1111/j.14697610.1987.tb00678.x 
Beardslee, W. R., Brent, D. A., Weersing, V. R., Clarke, G. N., Porta, G., Hollon, S. D., ... Garber, J. (2013). Prevention of depression in at-risk adolescents: Longer-term effects. JAMA Psychiatry, 70(11), 11611170. https://doi.org/10.1001/jamapsychiatry.2013.295

Beardslee, W. R., Versage, E. M., Wright, E. J., Salt, P., Rothberg, P. C., Drezner, K., \& Gladstone, T. R. (1997). Examination of preventive interventions for families with depression: evidence of change. Development and Psychopathology, 9(1), 109-130. https://doi.org/10.1017/S0954579497001090

Clarke, G. N., Hornbrook, M., Lynch, F., Polen, M., Gale, J., Beardslee, W. R., ... Seeley, J. (2001). A randomized trial of a group cognitive intervention for preventing depression in adolescent offspring of depressed parents. Archives of General Psychiatry, 58(12), 1127-1134.

https://doi.org/http://dx.doi.org/10.1001/archpsyc.58.12.1127

Claus, N., Marzano, L., Löchner, J., Starman, K., Voggt, A., Loy, F., ... Platt, B. (2019). Qualitative Evaluation of a Preventive Intervention for the Offspring of Parents with a History of Depression. BMC Psychiatry.

Compas, B. E., Forehand, R., Keller, G., Champion, J. E., Rakow, A., Reeslund, K. L., ... Cole, D. A. (2009). Randomized Controlled Trial of a Family Cognitive-Behavioral Preventive Intervention for Children of Depressed Parents. Journal of Consulting and Clinical Psychology. https://doi.org/10.1037/a0016930

Compas, B. E., Forehand, R., Thigpen, J. C., Keller, G., Hardcastle, E. J., Cole, D. A., ... Roberts, L. (2011). Family group cognitive-behavioral preventive intervention for families of depressed parents: 18- and 24month outcomes. Journal of Consulting and Clinical Psychology, 79(4), 488-499. https://doi.org/10.1037/a0024254

Compas, B. E., Forehand, R., Thigpen, J., Hardcastle, E., Garai, E., McKee, L., ... Sterba, S. (2015). Efficacy and moderators of a family group cognitive-behavioral preventive intervention for children of parents with depression. Journal of Consulting and Clinical Psychology. https://doi.org/10.1037/a0039053

Cracco, E., Goossens, L., \& Braet, C. (2017). Emotion regulation across childhood and adolescence: evidence for a maladaptive shift in adolescence. European Child and Adolescent Psychiatry, 26(8), 909921. https://doi.org/10.1007/s00787-017-0952-8

Cummings, E. M., \& Davies, P. T. (1994). Maternal Depression and Child Development. Journal of Child Psychology and Psychiatry, 35(1), 73-122. https://doi.org/10.1111/j.1469-7610.1994.tb01133.x

Dearing, K. F., \& Gotlib, I. H. (2009). Interpretation of ambiguous information in girls at risk for depression. Journal of Abnormal Child Psychology, 37(1), 79-91. https://doi.org/10.1007/s10802-008-9259-z

Döpfner, M., Berner, W., \& Lehmkuhl, G. (1994). Handbuch: Fragebogen für Jugendliche. Forschungsergebnisse zur deutschen Fassung der Youth Self-Report Form (YSR) der Child Behavior Checklist. 
Döpfner, M., Schmeck, K., \& Berner, W. (1994). Handbuch: Elternfragebogen über das Verhalten von Kindern und Jugendlichen. Forschungsergebnisse zur deutschen Fassung der Child Behavior Checklist (CBCL/4-18).

Downey, G., \& Coyne, J. C. (1990). Children of depressed parents: An integrative review. Psychological Bulletin, 108(1), 50-76. https://doi.org/10.1037/0033-2909.108.1.50

Dunbar, J. P., McKee, L., Rakow, A., Watson, K. H., Forehand, R., \& Compas, B. E. (2013). Coping, negative cognitive style and depressive symptoms in children of depressed parents. Cognitive Therapy and Research, 37(1), 18-28. https://doi.org/10.1007/s10608-012-9437-8

Fragebogen zur Erhebung der Emotionsregulation bei Kindern und Jugendlichen - FEEL-KJ. (n.d.).

Garber, J, Clarke, G. N., Weersing, V., Beardslee, W. R., Brent, D. A., Gladstone, T. R., ... lyengar, S. (2009). Prevention of depression in at-risk adolescents: a randomized controlled trial. JAMA Psychiatry, 301, 2215-2224.

Garber, Judy, Clarke, G. N., Weersing, V. R., Beardslee, W. R., Brent, D. A., Gladstone, T. R. G., ... Shamseddeen, W. (2009). Prevention of Depression in At-Risk Adolescents: A Randomized Controlled Trial. NIH Public Access, 301(21), 2215-2224. https://doi.org/10.1001/jama.2009.788.Prevention

Goodman, S. ., \& Gotlib, I. (1999). Risk for psychopathology in the children of depressed mothers: a developmental model for understanding mechanisms of transmission. Psychological Review, 106(3), 458-490.

Goodman, S. H., Rouse, M. H., Connell, A. M., Broth, M. R., Hall, C. M., \& Heyward, D. (2011). Maternal Depression and Child Psychopathology: A Meta-Analytic Review. Clinical Child An, 14(1), 1-27.

Hart, C. H., Newell, L., \& Olsen, S. (2003). Parenting skills and social-communicative competence in childhood. Handbook of Communication and Social Interaction Skill, 753-797.

Hautzinger, M., Bailer, M., Worall, H., \& Keller, F. (1994). Beck-Depressions-Inventar (BDI). German version. Test manual.(Bearbeitung der deutschen Ausgabe. Testhandbuch.). Göttingen: Huber.

Hayden, E. P., Hankin, B. L., Mackrell, S. V, Sheikh, H. I., Jordan, P. L., Dozois, D. J., ... Badanes, L. S. (2014). Parental depression and child cognitive vulnerability predict children's cortisol reactivity. Development and Psychopathology, 26(4 Pt 2), 1445-1460. https://doi.org/10.1017/s0954579414001138

Kovacs, M. (1992). The Children's Depression, Inventory (CDI). North Towanda, NY: Multi-Health System. https://doi.org/10.3724/SP.J.1041.2015.01004

Krohne, H. W., \& Pulsack, A. (1991). Erziehungsstil-Inventar. Goettingen: Beltz Test GmbH.

Kühner, C. (1997). Fragebogen zur Depressionsdiagnostik nach DSM-IV. 
Lenz, A. (2005). Vorstellungen der Kinder über die psychische Erkrankung ihrer Eltern. Eine explorative Studie. Praxis Der Kinderpsychologie Und Kinderpsychiatrie, 54(5), 382-398.

Loechner, J., Starman, K., Galuschka, K., Tamm, J., Schulte-Körne, G., Rubel, J., \& Platt, B. (2018).

Preventing depression in the offspring of parents with depression: A systematic review and meta-analysis of randomized controlled trials. Clinical Psychology Review, (November), 1-14.

https://doi.org/10.1016/j.cpr.2017.11.009

Lovejoy, M. C., Graczyk, P. A., O’Hare, E., \& Neuman, G. (2000). Maternal depression and parenting behavior: A meta-analytic review. Clinical Psychology Review, 20(5), 561-592.

https://doi.org/10.1016/S0272-7358(98)00100-7

Mason, W. A., Haggerty, K. P., Fleming, A. P., \& Casey-Goldstein, M. (2012). Family Intervention to Prevent Depression and Substance Use Among Adolescents of Depressed Parents. Journal of Child and Family Studies, 21(6), 891-905. https://doi.org/10.1007/s10826-011-9549-x

Platt, B, Pietsch, K., Krick, K., Oort, F., \& Schulte-Körne, G. (2014). Study protocol for a randomised controlled trial of a cognitive-behavioural prevention programme for the children of parents with depression: the PRODO trial. BMC Psychiatry, 14, 263.

Platt, Belinda, Pietsch, K., Krick, K., Oort, F., \& Schulte-Körne, G. (2014). Study protocol for a randomised controlled trial of a cognitive-behavioural prevention programme for the children of parents with depression: The PRODO trial. BMC Psychiatry, 14(1), 263. https://doi.org/10.1186/s12888-014-0263-2

Reck, C., Nonnenmacher, N., \& Zietlow, A. L. (2016). Intergenerational Transmission of Internalizing Behavior: The Role of Maternal Psychopathology, Child Responsiveness and Maternal Attachment Style Insecurity. Psychopathology, 49(4), 277-284. https://doi.org/10.1159/000446846

Sanford, M., Byrne, C., Williams, S., Atley, S., Ridley, T., Miller, J., \& Allin, H. (2003). A pilot study of a parenteducation group for families affected by depression. The Canadian Journal of Psychiatry, 48(2), 78-86. https://doi.org/10.1177/070674370304800203

Schäfer, J. Ö., Naumann, E., Holmes, E. A., Tuschen-Caffier, B., \& Samson, A. C. (2016). Emotion Regulation Strategies in Depressive and Anxiety Symptoms in Youth: A Meta-Analytic Review. Journal of Youth and Adolescence. https://doi.org/10.1007/s10964-016-0585-0

Schneider, S., \& Margraf, J. (2011). Diagnostic Interview for Psychiatric Disorders [Diagnostisches Interview bei psychischen Störungen]. Berlin: Springer.

Sfärlea, A., Löchner, J., Neumüller, J., Salemink, E., Asperud Thomsen, L., Starman, K., ... Platt, B. (2019). Passing on the half-empty glass: A transgenerational study of interpretation biases in children at risk for depression and their parents with depression. Journal of Abnormal Psychology, 128(2), 151-161. https://doi.org/10.1037/abn0000401 
Silk, J. S., Shaw, D. S., Forbes, E. E., Lane, T. L., \& Kovacs, M. (2006). Maternal depression and child internalizing: The moderating role of child emotion regulation. Journal of Clinical Child and Adolescent Psychology, 35(1), 116-126. https://doi.org/10.1207/s15374424jccp3501_10

Starman, K. (2018). Baseline differences and intervention effects of the "Gesund und Glücklich Aufwachsen (GUG-Auf)" prevention program for children of depressed parents. Retrieved from https://edoc.ub.uni-muenchen.de/22238/

Stieglitz, R.-D. (2002). Familientherapie aus verhaltenstherapeutischer Sicht. In Paar- und Familientherapie (pp. 121-135). Berlin, Heidelberg, New Yorl: Springer.

Stiensmeier-Pelster, J, Schürmann, M., Eckert, C., \& Pelster, A. (1994). Attributionsstil-Fragebogen für Kinder und Jugendliche (ASF-KJ). Handanweisung. Göttingen: Hogrefe.

Stiensmeier-Pelster, Joachim, Braune-Krickau, M., Schürmann, M., \& Duda, K. (2014). DIKJ. Depressionsinventar für Kinder und Jugendliche. Göttingen: Hogrefe.

Suppiger, A., In-Albon, T., Herren, C., Bader, K., Schneider, S., \& Margraf, J. (2008). Reliabilität des Diagnostischen Interviews bei Psychischen Störungen (DIPS für DSM-IV-TR) unter klinischen Routinebedingungen. Verhaltenstherapie, 18(4), 237-244. https://doi.org/10.1159/000169699

Tronick, E., \& Reck, C. (2009). Infants of depressed mothers. Harvard Review of Psychiatry, 17(2), 147156. https://doi.org/10.1080/10673220902899714

Unnewehr, S., Schneider, S., Margraf, J., Unnewehr, S., Margraf, J., Schneider, S., \& Margraf, J. (2009). Kinder-DIPS - Diagnostisches Interview bei psychischen Störungen im Kindes- und Jugendalter. 2. aktualisiterte und erweiterte Auflage. Heidelberg: Springer Medizin.

Weiß, R. H. (2006a). CFT 20-R. Grundintelligenztest Skala 2. Revision. . Göttingen: Hogrefe Verlag GmbH \& Co. KG.

Weiß, R. H. (2006b). Grundintelligenztest Skala 2 - Revision - (CFT 20-R). Göttingen: Hogrefe.

Weissman, M. ., Warner, V., Wickramaratne, P., Moreau, D., \& Olfson, M. (1997). Offspring of depressed parents. 10 Years later. Archives of General Psychiatry, 54(10), 932-940.

Weissman, M. M., Wickramaratne, P., Nomura, Y., Warner, V., Verdeli, H., Pilowsky, D. J., ... Bruder, G. (2005). Families at high and low risk for depression: A 3-generation study. Archives of General Psychiatry, 62(1), 29-36. https://doi.org/10.1001/archpsyc.62.1.29

Zietlow, A. L., Schlüter, M. K., Nonnenmacher, N., Müller, M., \& Reck, C. (2014). Maternal Self-confidence Postpartum and at Pre-school Age: The Role of Depression, Anxiety Disorders, Maternal Attachment Insecurity. Maternal and Child Health Journal, 18(8), 1873-1880. https://doi.org/10.1007/s10995-014$1431-1$ 


\section{Tables}

Table 1 Demographic and clinical characteristics, children and parents at baseline

\begin{tabular}{|c|c|c|c|c|}
\hline & EG & CG & Total & $p$-value \\
\hline Children & $\mathrm{n}=50$ & $\mathrm{n}=50$ & $N=100$ & \\
\hline Age, mean (SD) & $11.73(2.79)$ & $12.04(2.89)$ & $11.89(2.83)$ & .596 \\
\hline Gender (\%) female & 55.1 & 52.0 & 53.5 & .760 \\
\hline IQ, mean (SD) & $103.81(14.21)$ & $109.08(13.18)$ & $106.5(13.88)$ & .060 \\
\hline Siblings (\%) & 77.8 & 72.7 & 75.3 & .958 \\
\hline \multicolumn{5}{|l|}{ School type (\%) ${ }^{[1]}$} \\
\hline Primary school & 31.0 & 34.1 & 32.5 & .822 \\
\hline Hauptschule & 4.8 & 2.4 & 3.8 & - \\
\hline Realschule & 14.3 & 9.8 & 12.0 & - \\
\hline Gymnasium & 47.6 & 51.2 & 49.4 & .839 \\
\hline Parents & $\mathrm{n}=50$ & $\mathrm{n}=50$ & $\mathbf{N}=100$ & \\
\hline Age, mean (SD) & $45.15(5.80)$ & $47.10(7.01)$ & $46.06(6.43)$ & 157 \\
\hline Gender (\%) female & 60.0 & 62.7 & 61.4 & .684 \\
\hline Highest level of education (\%) & & & & .143 \\
\hline High school & 14.0 & 18.2 & 15.8 & \\
\hline A-levels & 23.3 & 30.3 & 26.3 & \\
\hline University & 46.5 & 51.5 & 48.7 & \\
\hline Doctoral degree & 16.3 & 0 & 9.2 & \\
\hline Family income (\%) & & & & .704 \\
\hline$-2000 € /$ month & 10.3 & 12.5 & 11.3 & \\
\hline $2000-3000 € /$ month & 17.9 & 18.8 & 18.3 & \\
\hline $3000-4000 € /$ month & 15.4 & 18.8 & 16.9 & \\
\hline $4000-5000 € /$ month & 30.8 & 25.0 & 28.2 & \\
\hline$>5000 € /$ month & 25.6 & 25.0 & 25.4 & \\
\hline Depressive Symptoms (BDI-II) & $16.7(10.04)$ & 17.7 (12.29) & $17.20(11.10)$ & .620 \\
\hline Currently depressed (\%) & 58.0 & 56.9 & 57.4 & .421 \\
\hline \multicolumn{5}{|l|}{ Treatment experience (\%) } \\
\hline Psychotherapy & 92.3 & 94.3 & 93.2 & .504 \\
\hline Psychopharmaceuticals & 82.1 & 69.7 & 76.4 & .165 \\
\hline
\end{tabular}

Note. BDI-II = Beck's Depression Inventory

Table 2 Instruments used to measure eligibility criteria and outcome variables 


\begin{tabular}{|c|c|c|}
\hline & Measure & Instrument \\
\hline \multirow[t]{5}{*}{ Eligibility criteria } & Diagnostic status (child) & $\begin{array}{l}\text { K-DIPS psychiatric } \\
\text { interview }\end{array}$ \\
\hline & Intelligence (child) & CFT 20-R test \\
\hline & Diagnostic status (parent) & $\begin{array}{l}\text { DIPS psychiatric } \\
\text { interview }\end{array}$ \\
\hline & Personality disorder (parent) & SKID II questionnaire \\
\hline & Psychopathology ( $2^{\text {nd }}$ parent) & $\begin{array}{l}\text { SCL-90-R } \\
\text { questionnaire }\end{array}$ \\
\hline \multirow{2}{*}{$\begin{array}{l}\text { Main outcome } \\
\text { variables }\end{array}$} & Depressive symptoms (child) & DIKJ questionnaire \\
\hline & $\begin{array}{l}\text { Internalising and externalising symptoms (self- } \\
\text { and parent-report) }\end{array}$ & $\begin{array}{l}\text { YSR, CBCL } \\
\text { questionnaire }\end{array}$ \\
\hline \multirow{4}{*}{$\begin{array}{l}\text { Secondary } \\
\text { outcome } \\
\text { variables }\end{array}$} & ER (child) & $\begin{array}{l}\text { FEEL-KJ } \\
\text { questionnaire }\end{array}$ \\
\hline & Attributional style (child) & ASF questionnaire \\
\hline & Parenting style (child) & ESI questionnaire \\
\hline & Knowledge of depression (child) & Questionnaire \\
\hline & BDI-II questionnaire \\
\hline \multicolumn{3}{|c|}{$\begin{array}{l}\text { Note. ASF = Attributionsstil-Fragebogen für Kinder und Jugendliche; BDI-II = Beck's } \\
\text { Depression Inventory; CASE = Child and Adolescent Survey of Experiences, child and parent } \\
\text { version; CBCL = Child Behaviour Checklist; CFT 20-R = Culture Fair Test 20. Revision; DIKJ } \\
\text { = Depressions-Inventar für Kinder und Jugendliche; DIPS = Diagnostisches Interview bei } \\
\text { psychischen Störungen; ER = Emotion Regulation; ESI = Erziehungsstil-Inventar; FEEL-KJ = } \\
\text { Fragebogen zur Erhebung der Emotionsregulation bei Kindern und Jugendlichen; K-DIPS = } \\
\text { Diagnostisches Interview bei psychischen Störungen im Kindes- und Jugendalter; SCL-90-R = } \\
\text { Symptom Checklist; SKID II = Strukturiertes Klinisches Interview für DSM-IV; YSR = Youth } \\
\text { Self-Report. }\end{array}$} \\
\hline
\end{tabular}

Table 3 Statistical comparison of outcome variables (raw means, SD) at baseline and postassessment 


\begin{tabular}{|c|c|c|c|c|c|c|c|c|}
\hline Main outcome variables & EG & CG & EG & CG & F & $\mathrm{df}$ & $p$ & $\eta \mathrm{p} \square$ \\
\hline Depressive symptoms & $\begin{array}{l}8.24 \\
(5.60)\end{array}$ & $\begin{array}{l}7.49 \\
(3.91)\end{array}$ & $\begin{array}{l}8.28 \\
(6.45)\end{array}$ & $\begin{array}{l}5.67 \\
(4.05)\end{array}$ & 2.49 & $\begin{array}{l}1 \\
98\end{array}$ & .118 & 0.02 \\
\hline $\begin{array}{l}\text { Internalising symptoms } \\
\text { (self-report) }\end{array}$ & $\begin{array}{l}9.99 \\
(7.34)\end{array}$ & $\begin{array}{l}7.96 \\
(5.34)\end{array}$ & $\begin{array}{l}7.95 \\
(6.90)\end{array}$ & $\begin{array}{l}8.02 \\
(5.24)\end{array}$ & 5.42 & $\begin{array}{l}1 \\
98\end{array}$ & .022 & 0.05 \\
\hline $\begin{array}{l}\text { Externalising symptoms } \\
\text { (self-report) }\end{array}$ & $\begin{array}{l}9.03 \\
(5.29)\end{array}$ & $\begin{array}{l}8.73 \\
(5.76)\end{array}$ & $\begin{array}{l}7.82 \\
(5.84)\end{array}$ & $\begin{array}{l}10.42 \\
(4.97)\end{array}$ & 8.44 & $\begin{array}{l}1 \\
98\end{array}$ & .005 & 0.08 \\
\hline $\begin{array}{l}\text { Depression/anxiety (self- } \\
\text { report) }\end{array}$ & $\begin{array}{l}4.98 \\
(3.18)\end{array}$ & $\begin{array}{l}3.51 \\
(2.56)\end{array}$ & $\begin{array}{l}3.03 \\
(3.97)\end{array}$ & $\begin{array}{l}4.43 \\
(3.12)\end{array}$ & 8.54 & 1,98 & .004 & 0.08 \\
\hline $\begin{array}{l}\text { Internalising symptoms } \\
\text { (parent-report) }\end{array}$ & $\begin{array}{l}9.01 \\
(6.15)\end{array}$ & $\begin{array}{l}8.97 \\
(5.45)\end{array}$ & $\begin{array}{l}7.22 \\
(7.51)\end{array}$ & $\begin{array}{l}8.14 \\
(5.49)\end{array}$ & 0.76 & $\begin{array}{l}1 \\
98\end{array}$ & .384 & 0.001 \\
\hline $\begin{array}{l}\text { Externalising symptoms } \\
\text { (parent-report) }\end{array}$ & $\begin{array}{l}7.17 \\
(5.35)\end{array}$ & $\begin{array}{l}6.34 \\
(5.61)\end{array}$ & $\begin{array}{l}5.59 \\
(5.43)\end{array}$ & $\begin{array}{l}4.41 \\
(3.68)\end{array}$ & 0.88 & $\begin{array}{l}1 \\
98\end{array}$ & .767 & 0.001 \\
\hline $\begin{array}{l}\text { Depression/anxiety } \\
\text { (parent-report) } \\
\text { Potential mechanisms } \\
\end{array}$ & $\begin{array}{l}4.48 \\
(3.60)\end{array}$ & $\begin{array}{l}4.06 \\
(3.14)\end{array}$ & $\begin{array}{l}3.46 \\
(3.92)\end{array}$ & $\begin{array}{l}3.71 \\
(2.83)\end{array}$ & 0.96 & $\begin{array}{l}1 \\
98\end{array}$ & .328 & 0.001 \\
\hline Adaptive ER & $\begin{array}{l}128.46 \\
(26.91)\end{array}$ & $\begin{array}{l}133.73 \\
(26.37)\end{array}$ & $\begin{array}{l}122.71 \\
(35.34)\end{array}$ & $\begin{array}{l}136.23 \\
(24.45)\end{array}$ & 1.89 & $\begin{array}{l}1 \\
98\end{array}$ & .172 & 0.002 \\
\hline Maladaptive $E R$ & $\begin{array}{l}71.22 \\
(13.02)\end{array}$ & $\begin{array}{l}68.34 \\
(14.21)\end{array}$ & $\begin{array}{l}70.61 \\
(14.15)\end{array}$ & $\begin{array}{l}70.14 \\
(11.08)\end{array}$ & 0.76 & $\begin{array}{l}1 \\
98\end{array}$ & .383 & 0.001 \\
\hline $\begin{array}{l}\text { Positive attributional } \\
\text { style }\end{array}$ & $\begin{array}{l}66.06 \\
(9.27)\end{array}$ & $\begin{array}{l}65.66 \\
(9.67)\end{array}$ & $\begin{array}{l}69.21 \\
(8.52)\end{array}$ & $\begin{array}{l}70.03 \\
(6.98)\end{array}$ & 0.51 & $\begin{array}{l}1 \\
98\end{array}$ & .477 & 0.005 \\
\hline $\begin{array}{l}\text { Negative attributional } \\
\text { style }\end{array}$ & $\begin{array}{l}59.66 \\
(9.49)\end{array}$ & $\begin{array}{l}58.78 \\
(10.83)\end{array}$ & $\begin{array}{l}65.94 \\
(8.57)\end{array}$ & $\begin{array}{l}63.69 \\
(8.08)\end{array}$ & 0.54 & $\begin{array}{l}1 \\
98\end{array}$ & .465 & 0.005 \\
\hline Positive parenting & $\begin{array}{l}75.00 \\
(9.48)\end{array}$ & $\begin{array}{l}69.57 \\
(10.21)\end{array}$ & $\begin{array}{l}74.22 \\
(12.68)\end{array}$ & $\begin{array}{l}71.22 \\
(11.40)\end{array}$ & 0.96 & $\begin{array}{l}1 \\
98\end{array}$ & .330 & 0.010 \\
\hline Negative parenting & $\begin{array}{l}67.19 \\
(12.52)\end{array}$ & $\begin{array}{l}67.05 \\
(9.63)\end{array}$ & $\begin{array}{l}63.67 \\
(10.41)\end{array}$ & $\begin{array}{l}66.63 \\
(7.52)\end{array}$ & 1.86 & $\begin{array}{l}1 \\
98\end{array}$ & .175 & 0.019 \\
\hline Knowledge of depression & $\begin{array}{l}33.36 \\
(4.26)\end{array}$ & $\begin{array}{l}32.03 \\
(2.99)\end{array}$ & $\begin{array}{l}35.79 \\
(2.40)\end{array}$ & $\begin{array}{l}34.74 \\
(2.98)\end{array}$ & 0.14 & 1,98 & .704 & 0.001 \\
\hline
\end{tabular}

Potential moderator

\begin{tabular}{|c|c|c|c|c|c|}
\hline Parental depression & $\begin{array}{l}16.70 \\
(9.84)\end{array}$ & $\begin{array}{l}17.76 \\
(11.25)\end{array}$ & $\begin{array}{l}11.96 \\
(8.98)\end{array}$ & $\begin{array}{l}13.92 \\
(7.99)\end{array}$ & $\begin{array}{llll}0.17 & 1,98 & 677 & 0.002\end{array}$ \\
\hline
\end{tabular}

Note. ASF $=$ Attributionsstil-Fragebogen für Kinder und Jugendliche; BDI-II $=$ Beck's Depression Inventory; CBCL = Child Behaviour Checklist; DIKJ = Depressions-Inventar für 
Kinder und Jugendliche; ER = Emotion Regulation; ESI = Erziehungsstil-Inventar; FEEL-KJ = Fragebogen zur Erhebung der Emotionsregulation bei Kindern und Jugendlichen; YSR = Youth Self-Report.

[1] German secondary schools are categorised into three levels based on achievement at the end of primary school (increasing with grades): Hauptschule, Realschule or Gymnasium.

\section{Figures}




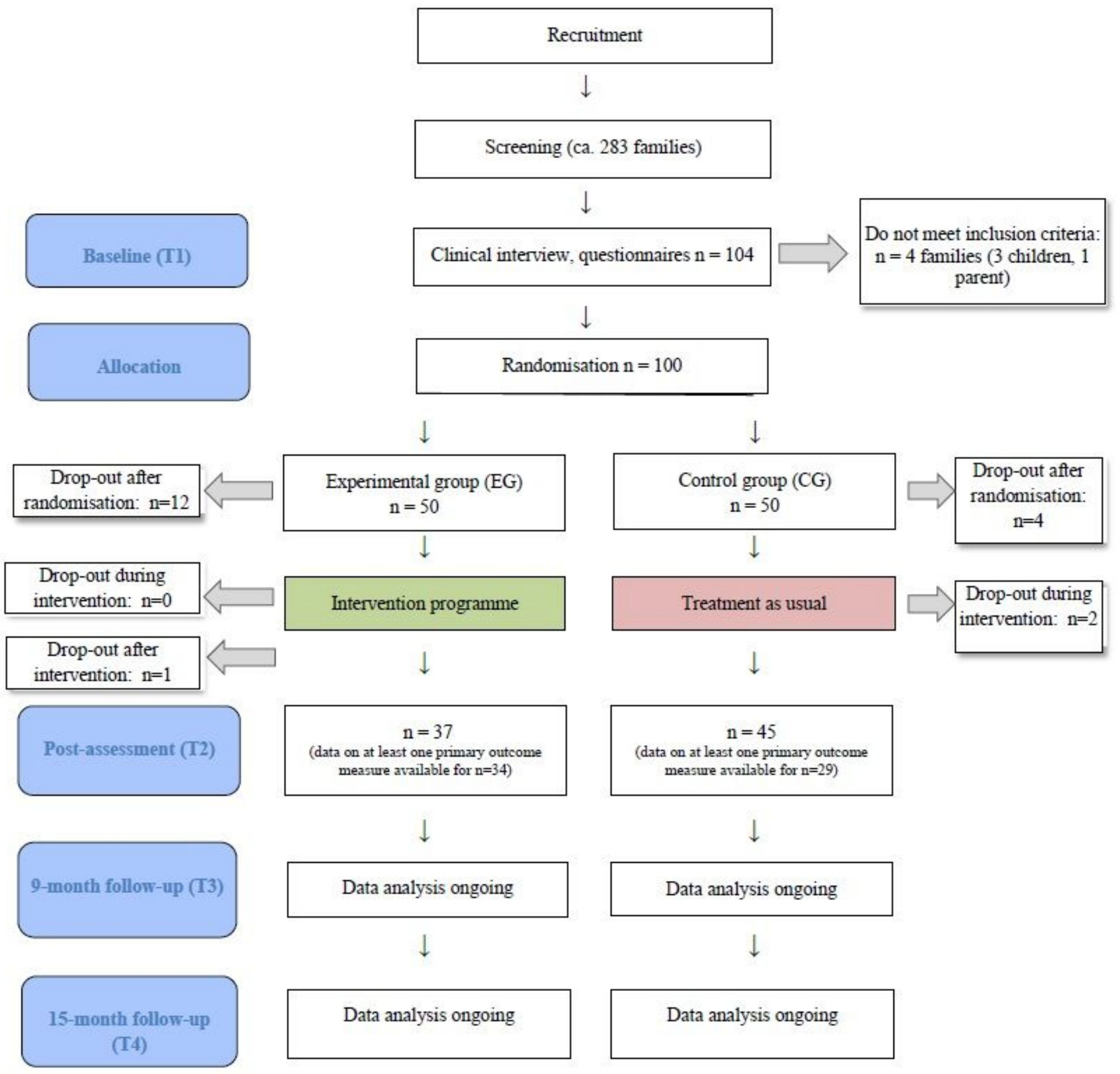

\section{Figure 1}

Participant flow/study design

\section{Supplementary Files}

This is a list of supplementary files associated with this preprint. Click to download. 
- Supplement.docx

Page $32 / 32$ 